\title{
Variations
}

Variations

Revue internationale de théorie critique

19 | 2016

Critique des humanités numériques

\section{Simulation sociale et simulacre structural}

\section{Arthur Lefèvre}

\section{OpenEdition}

Journals

Édition électronique

URL : http://journals.openedition.org/variations/720

DOI : 10.4000/variations. 720

ISSN : 1968-3960

\section{Éditeur}

Les amis de Variations

\section{Référence électronique}

Arthur Lefèvre, «Simulation sociale et simulacre structural », Variations [En ligne], 19 | 2016, mis en ligne le 06 avril 2016, consulté le 30 avril 2019. URL : http://journals.openedition.org/variations/720 ; DOI : 10.4000/variations.720

Ce document a été généré automatiquement le 30 avril 2019

Les ami•e•s de Variations 


\title{
Simulation sociale et simulacre structural
}

\author{
Arthur Lefèvre
}

1 La simulation sociale est un domaine de recherche dont les principes explicites sont la formalisation informatique des processus sociaux. Il s'agit ici d'interroger cette méthode d'«expérience de pensée» assistée par informatique, où des agents «autonomes, hétérogènes, et à la rationalité limitée en interactions locales dans un espace défini » (Epstein, 1999) reproduisent des phénomènes dynamiques dits «bottom-up». En éclairant, ici, l'histoire et les ambitions de la simulation sociale, nous cherchons à montrer comment ce domaine encore jeune, organisé principalement autour du Journal of artificial societies and social simulation (JASSS) ${ }^{1}$, fonctionne et conçoit son travail. Nous pointerons certains impensés qui sous-tendent en silence ses implémentations, hors des débats épistémologiques qui agitent le champ.

\section{La simulation sociale, une omni-disciplinarité ?}

2 La simulation sociale est un champ de recherche aux contours extrêmement flous, dont les développements dans les « humanités numériques » (HN) sont une manifestation de la fluidité de ce domaine qui se veut fondamentalement interdisciplinaire voire «noncompartimentale » (Conte et al., 2012).

\section{Des ambitions frustrées}

3 Depuis les travaux de simulation sur les populations Anasazis² (Dean et al., 2000) qui ont suscité de nombreux espoirs quant aux possibilités ouvertes par la simulation sociale, celle-ci a attiré un intérêt assez large (numéros spéciaux de l'American review of sociology, des Proceedings of national academy of sciences, etc.) qui lui a permis de s'installer, sur la durée, dans le paysage de la recherche et d'y développer ses propres problématiques. Et pourtant, avec le recul de ces dernières années, «même le partisan le plus enthousiaste ne pourrait pas soutenir que les modèles multi-agents aient dramatiquement changé le 
paysage des sciences sociales actuelles » (Squazonni 2010). C'est que la simulation sociale intéresse bien davantage les sciences informatiques, physiques et naturelles que les sciences humaines et sociales ${ }^{3}$ (Squazonni, Casnici, 2013). Il est à ce titre assez instructif de noter qu'en 2014, la revue française de sociologie publie un numéro d'introduction à la simulation multi-agent (Manzo, 2014) : si la simulation sociale se voit comme un lieu du dialogue interdisciplinaire, celui-ci est encore clairement déséquilibré et les chercheurs du domaine le déplorent volontiers.

4 Les sciences sociales sont pourtant bien au cœur des ambitions de légitimation de la simulation, qui s'expriment sous deux formes et dessinent les avenirs possibles quant à leurs relations avec celles-ci. Une première version formule un projet que l'on qualifiera volontiers de conquérant : « les sciences sociales en général et la sociologie en particulier subiraient un changement de paradigme dramatique, découlant de l'incorporation de la méthode scientifique des sciences physiques " (Conte et al., 2012). Il s'agit bien de transformer les sciences sociales, de les renouveler intégralement, passant outre l'histoire, les questionnements et les problématiques du champ qu'ils prétendent renouveler sur le mode de la table rase, parce qu'ils en ignorent presque tout et les perçoivent essentiellement comme pré-scientifiques, manquant de méthode (Moss, Edmonds, 2005). Quoique l'on puisse arguer qu'il s'agit là d'une fanfaronnade d'un champ de recherche encore jeune, les moyens alloués ou réclamés ${ }^{4}$ ne peuvent qu'alerter les sciences sociales sur une tentative d'annexion pure et simple par des chercheurs issus de sciences «dures». La seconde version, clairement pragmatique, souhaite plus modestement ajouter la simulation multi-agent dans «la boîte à outil commune des chercheurs en sciences sociales» (Waldher, Wijermans, 2013), aux côtés des outils statistiques ordinaires. Cela explique pourquoi la démarcation explicite la plus récurrente de la simulation, la distinction qui lui permet de se dégager comme champ autonome, est formulée vis-à-vis des méthodes mathématiques, statistiques et équations («qu'est-ce que ça apporte de plus, de différent?»). L'agent-based modeling est opposé à l'equation based-modeling (Cecconi et al., 2010), parce que l'outil a encore besoin de faire ses preuves en tant que tel : la méthode elle-même est remise en question, et non ce qu'elle apporte et dans quelle situation. La simulation sociale est encore trop jeune pour être divisée en écoles explicitement divergentes, mais on ne peut prendre ces ambitions comme un continuum parfait, bien qu'une ambiguïté importante subsiste: si la simulation n'est qu'un outil au même titre que les techniques statistiques, cet outil porte des conceptions (nécessairement conquérantes) qu'il s'agit d'éclairer pour en comprendre les enjeux critiques.

\section{Une science dure}

Comme nous l'avons mentionné supra, les trois domaines les plus intéressés par la simulation sociale sont l'informatique, les sciences physiques et les sciences naturelles, ce qui souligne l'importance des liens historiques et des circulations scientifiques entre ces champs. Les sciences informatiques citent volontiers des travaux de simulation sociale pour des raisons évidentes liées au support de la simulation, ou plus prosaïquement sans doute, parce que les recherches en simulation sociale sont d'abord le fait de chercheurs en informatique. Mais plus récemment, la recherche en intelligence artificielle, qui pour résoudre des problèmes peut recourir à des algorithmes dits distribués (c'est-à-dire fragmentés en unités autonomes en interactions), s'inspire volontiers de processus 
sociaux, après avoir exploré l'évolution biologique ou la neurologie. Plus surprenant peut-être, les sciences physiques s'intéressent également énormément à la simulation sociale, au point que le manifeste pour une science sociale informatique est publié dans l' European physical journal, et non dans le JASSS, comme on pourrait s'y attendre. C'est que l'histoire de la simulation informatique commence par des applications pour la physique, et que l'habitude mathématique a conduit à penser les systèmes abstraitement. Une fois un modèle créé pour une question, il est possible de l'abstraire de ses caractéristiques « locales » puis de le réutiliser ou de l'adapter à une autre situation, parfois très éloignée. C'est ainsi qu'on peut comprendre l'émergence de travaux de sociophysique (Galam, 2012), qui cherchent à recycler des modèles abstraits issus de la physique pour expliquer des phénomènes sociaux.

6 La simulation sociale entretient des liens encore plus profonds avec la simulation biologique et ce à de nombreux niveaux. Les premières simulations de vie artificielle, telles que proposées par John Conway (Gardner, 1970), sont appuyées sur des automates cellulaires aux règles extrêmement simples mais pouvant produire de très nombreux phénomènes d'auto-reproduction. Ce sont les mêmes automates cellulaires qui ont inspiré les premières simulations sociales, dont les travaux fondateurs de Thomas Schelling (Schelling, 1971). De plus, la simulation biologique s'est beaucoup intéressée à la socialité animale, aux insectes sociaux notamment, qui produisent des comportements collectifs très complexes à partir de comportements individuels très simples (Drogoul, 1993). Sur un mode plus abstrait encore, la théorie des jeux a permis l'ouverture d'une série de simulations transversales sur les conditions d'émergence et d'évolution de la coopération entre agents autonomes, dont la littérature de la simulation sociale est très friande depuis les travaux fondateurs de Robert Axelrod (Axelrod, 1984), et a conduit à des projets d'études davantage habillés de biologie, mais dont les préoccupations sont strictement similaires, comme le projet $\mathrm{EACH}^{5}$ (Centola et al., 2000). Enfin, les simulations socio-environnementales resserrent encore les liens entre la simulation sociale et les sciences naturelles, quoique sur un mode un peu différent. La simulation qui a attiré l'attention sur la simulation sociale (Dean et al., 2000), par exemple, a reproduit l'implantation des populations dans une vallée mexicaine précolombienne, sous le contrôle croisé de données archéologiques et environnementales. Il s'agit moins ici de construire un parallèle entre les sociabilités humaines et animales que de placer les sociétés dans leur conditions naturelles. On comprend, dans ces conditions, que les concepts, méthodes et préoccupations de la simulation sociale soient intimement liés à ceux des sciences « dures ». Ainsi, l'introduction de la simulation sociale dans les sciences humaines, alors même que leurs questionnements respectifs sont étrangers les uns aux autres, ne peut qu'être source de confusion.

\section{Quiproquos et acclimatation}

7 Le fait que les sciences économiques se soient davantage emparées de la simulation s'inscrit dans une tendances de longue haleine de «durcissement» par la mathématisation de l'économie orthodoxe. Ces écoles dominantes souhaitent au fond être la plus «scientifique» des sciences sociales, prenant les outils mathématiques des sciences exactes pour le gage de scientificité qu'il lui manquerait. La simulation sociale devient ainsi un nouvel instrument de «scientificisation» du domaine, au prix d'un double malentendu. 
8 En premier lieu, les conflits théoriques, épistémiques et méthodologiques font notoirement rage en économie (Orléan, 2015), comme dans toutes les sciences sociales, alors que la simulation se trouve dans ces conflits comme une parfaite étrangère. Si l'on trouve parfois mention de l'individualisme méthodologique (Macy, Flache, 2009), la règle du domaine est plutôt un agnosticisme « technopragmatique » (Granjon, 2015). Les débats théoriques sans visées pratiques y sont perçus comme "abscons", "peu utiles », et mettant en cause la «neutralité scientifique » (Rossiter et al., 2010). La forme la plus pure en est sans doute la formule prêtée à un "réaliste », discutant avec un «rêveur » et un « expérimentaliste » (Deffuant et al., 2009) : «Locke, Hume, Smith [...] 250 ans plus tard, toutes ces magnifiques théories n'intéressent qu'une douzaine de spécialistes européens décadents. K. Marx et ses continuateurs. [...] Que reste-t-il de ces dramatiques délires théoriques? Des ruines et la mort. Les recherches en sciences sociales les plus récentes, quoique leurs prétentions soient plus modestes, ne vont pas moins dans une mauvaise direction ». Et de conclure : "[les simulations] peuvent être "dirigées par la réalité" et ignorer toutes ces illusions théoriques. Une nouvelle science, oui, mais une "tabula rasa", qui s'en tient à des cas d'étude réels!». Quoique le trait soit volontairement exagéré par les auteurs de ces dialogues fictifs aux accents théâtraux, il n'en reste pas moins que le rejet de la pensée sociologique préexistante n'est jamais mis en question par ses contradicteurs, et que le débat se concentre exclusivement sur les conditions de possibilité d'une « nouvelle » théorie basée sur de « nouvelles » pratiques.

9 Ainsi, la simulation sociale arrive désarmée dans des débats qu'elle perçoit comme n'étant pas les siens, mettant ses moyens au service de quiconque lui accordera la légitimité qu'elle cherche au sein des sciences sociales, c'est-à-dire, ironiquement, principalement ceux qui prétendent les quitter. En retour de cet aveuglement, la simulation se trouve identifiée à l'école qui s'en est emparée la première, voyant dans cet outil «neutre » un moyen de refonder l'individualisme méthodologique en amendant l' homo economicus chancelant d'une rationalité et d'informations limitées. Cependant, aucun outil ne saurait être neutre, et nous tenterons de démontrer que la philosophie intégrée à la simulation sociale n'est pas l'individualisme méthodologique, et que, si les chercheurs en simulation ne sont clairement pas conscients de ce qu'ils font pourtant, on peut la mettre au jour à la condition de savoir lire entre ses lignes de code.

\section{Modéliser}

10 Construire une simulation informatique d'un phénomène implique d'en produire un modèle formel, c'est la vertu principale qu'identifie Joshua Epstein à propos de la simulation: expliciter des modèles pour en permettre la discussion (Epstein, 2008). De plus, pour parler de simulation, il faut encore que ce modèle soit dynamique et distribué. Le comportement du système représentant le phénomène est observé selon ce qui en émerge, c'est-à-dire selon ce que le système devient par l'action de ses sous-parties autonomes (Baker, 2010).

\section{Ce que simuler veut dire}

11 Le principe central de la simulation, l'émergence, ne prend sens que dans une démarche scientifique plus large: produire une explication générative suffisante du phénomène simulé (Epstein, 1999). L'explication est dite générative parce qu'il s'agit de produire des 
résultats macros (à l'échelle du système) à partir de comportements micros (à l'échelle des agents), donc d'expliquer les premiers par les seconds. Et l'explication est dite suffisante parce que le même phénomène peut aussi bien admettre une explication générative concurrente, c'est-à-dire au sens le plus mathématique possible, que l'explication est suffisante mais pas nécessaire. Nous décrivons ici, pour exemple, une des simulations les plus largement reconnues comme étant aux origines de la simulation sociale (Schelling, 1971), qui propose une simulation de ségrégation à partir d'automates cellulaires. Celle-ci porte déjà une grande part des problématiques de la simulation sociale, c'est pourquoi elle est décrite en détail et resservira de base à des discussions ultérieures. Le travail original de Schelling a été simulé sur papier mais Uri Wilenski en a proposé une version informatique implémentée en NetLogo qui est la version que nous décrivons ici (Wilenski 1997). Cette simulation fonctionne comme suit :

- Des agents de deux types (Rouge et Vert), en proportions équivalentes, sont placés sur une grille de taille fixe. Il y a un agent par case au maximum, et la proportion de cases laissées libres est une variable, laissée aux mains du simulateur qui peut la faire varier avant le lancement de la simulation ;

- À chaque tour, chaque agent regarde les huit cases voisines et compte les agents partageant son identité et ceux ne la partageant pas (les cases libres sont ignorées). Si la proportion d'agents qui lui sont similaires par rapport à ceux qui lui sont différents est inférieure à un taux minimal, l'agent est insatisfait ;

- Le taux qui détermine la satisfaction est le même pour tous les agents, qui réagissent donc selon la même règle à des voisinages différents ;

- À chaque fin de tour, les agents insatisfaits déménagent vers une case déjà libre ou laissée libre par un autre agent insatisfait ;

- La simulation prend fin (on dira qu'elle converge) si tous les agents sont satisfaits de leur voisinage.

12 Le phénomène macroscopique étudié est ici la formation d'espaces ségrégués au sein d'une société. Le comportement microscopique est un désir quantifié de non-mixité minimale, associé à un déplacement en cas d'insatisfaction. Le principe de l'explication générative que l'expérience répétée permet de tirer est que des taux relativement faibles de désir de non-mixité suffisent à produire des sociétés très ségréguées. Avant d'approfondir la question de l'interprétation d'une simulation, nous souhaitons répondre à une objection ici lancinante, mais adressée à de très nombreux travaux de simulation : "c'est trop simple » ou « il manque tel paramètre » (Waldher, Wijermans, 2013). Si cette objection est souvent fondée et permet en retour l'affinement des simulations, elle est souvent elle-même trop simple et mérite d'être raffinée pour gagner en pertinence.

\section{Simplifier}

13 Tout modèle, et a fortiori toute simulation, est nécessairement une simplification, une abstraction formelle. "Qu'est-ce que l'abstraction ici? Ce n'est pas simplement la généralisation [...] Ce n'est pas un raisonnement par métaphores ou analogies non plus [... ] L'abstraction est une façon de penser où "de nouvelles idées ou concepts sont formés en considérant plusieurs objets et dont sont omises les caractéristiques qui les distinguent les uns des autres" (Rosen, 2014) L'abstraction signifie rejeter les détails, se débarrasser du particulier» (Healy, 2015). Pour ce qui concerne la simulation de Schelling, il est évident qu'un grand nombre de détails ont été passés par-dessus bord : les identités sont 
binaires et exclusives, les deux populations sont équivalentes en nombre, la grille de voisinage est parfaitement carrée, le déplacement ne pose aucune contrainte, les agents obéissent tous à la même règle, etc. La critique du simplisme de la simulation peut conduire à des raffinements qui ne mettent pas en cause les fondements du modèle initial: l'ajout de contraintes économiques sur le déplacement (Benard, Willer, 2007), déséquilibrer les proportions entre populations (Hatna, Benenson, 2012), différencier individuellement les préférences (Xie, Zhou, 2012), etc. Le pivot de la critique d'une simulation sociale tient ainsi dans l'analyse de ses niveaux d'abstractions, et des manques que ceux-ci présentent vis-à-vis d'une situation empirique, ou du moins, d'une abstraction moins élevée d'une situation empirique.

La simulation la plus abstraite possible serait sans doute l'étude de Robert Axelrod sur le dilemme du prisonnier itéré (Axelrod, 1984). Ce dilemme est directement issu de la théorie des jeux : Deux prisonniers qui ne peuvent communiquer doivent choisir de trahir ou ne pas trahir l'autre, en le dénonçant:

- Si aucun ne parle, les deux prisonniers subissent une peine minime ;

- Si les deux trahissent, les deux subissent une peine lourde ;

- Si un seul des deux trahit, il est libéré et celui qui est trahi subit une peine encore plus lourde que si les deux avaient trahi.

La théorie des jeux a établi que ce jeu possède deux équilibres, la double trahison et la double collaboration, et que la seule solution raisonnable sur un jeu unique est de trahir, bien que cela conduise à l'équilibre le moins satisfaisant. Cependant, le dilemme du prisonnier itéré pose la question de l'émergence de la coopération en permettant d'établir des stratégies temporelles. La coopération/trahison sont ici des phénomènes pris à un niveau d'abstraction très élevé qui n'est pas mis en question en tant que tel parce que pouvant représenter de très nombreuses formes d'interactions impliquant la (non-)réciprocité. La critique de son simplisme a donc porté sur le réalisme de l'espace social. Ainsi, des travaux de topologies des relations sociales et de ses effets sur l'émergence de la coopération ont été régulièrement proposés (Seltzer, Smirnov, 2015). Par ailleurs, la question des stratégies collectives a été mise au jour, par un système de labels qui permet l'identification progressive et l'ostracisme (la trahison systématique) des free-riders (Shutter, Hales, 2013), ou l'étude de la domination évolutive de stratégies ethnocentristes (Hartshorn, 2013) ou culturelles (Hales, Edmonds, 2015).

À l'autre bout du spectre, on trouverait des simulations extrêmement précises, pleines de détails complexes comme l'expérience fondatrice des Anasazis artificiels ou, plus ambitieuses encore, NewTies (Gilbert et al., 2006). Cette dernière propose rien moins que de se livrer à une explication générative de l'émergence du langage. Les agents y sont dotés de caractéristiques physiques génétiques sujettes à la mutation entre générations, et d'un système d'apprentissage leur permettant d'échanger des informations. De plus, ceux-ci sont plongés dans des mondes naturels divers, desquels l'extraction de ressources est nécessaire pour leur survie et celle de leur descendance, à qui il faut encore enseigner leurs connaissances. Enfin, ils sont capables d'échanger des ressources, ce qui devait permettre par exemple d'établir l'émergence du potlach ou d'accords collectifs sur la collecte des ressources permettant leur bon renouvellement. À notre connaissance, cette expérience, malgré des fonds très importants de l'Union européenne, n'a pas abouti et a été abandonnée, malheureusement sans en publier de bilan. Comme le note Guillaume Deffuant, «les modèles complexes ne sont pas nécessairement plus réalistes que les modèles simples », puisqu'« il est très facile de produire des simulations complexes. La 
partie difficile est d'établir des liens forts entre leurs propriétés dynamiques et des résultats de la psychologie ou de la sociologie » (Deffuant, 2003). Ce que nous amenderons tout de même : produire des simulations complexes est déjà très difficile, l'établissement de liens forts avec des résultats empiriques ajoutant encore un surcroit de difficultés.

\section{Omettre}

La critique de la simulation doit savoir établir le degré d'abstraction auquel se situe une simulation pour en critiquer adéquatement les manques. Toutefois, si l'abstraction est une nécessité de tout modèle, il faut pourtant clairement différencier ce qui est de l'ordre de la simplification nécessaire à ce degré d'abstraction, qui conduit à une critique sur le mode de l'affinement, et ce qui relève des amputations graves de ce qui est prétendument modélisé, qui en changent le sens par des présupposés impensés. Les études explicitement politiques ou économiques sont sans doute les plus sensibles à ce genre de troncatures.

Dans une étude du comportement des partis socio-démocrates européens (Schumacher, Vis, 2012), il est par exemple explicitement posé que l'économie est une variable d'ordre environnemental pour la prise de décision politique, qui n'a en retour aucun effet sur l'économie. Dans cette simulation, les partis politiques sont des agents cherchant à être élus par d'autres agents, les électeurs, et dont la politique centrale est l'avancement ou le retrait de l'«État-providence». Si l'étude montre clairement que les partis sociodémocrates se droitisent davantage lorsqu'ils sont plus attirés par l'opinion de l'électeur moyen que par l'opinion de leurs propres électeurs, les partis n'ont en fait aucune forme de pouvoir sur ce qui est considéré comme les variables majeures de formation des opinions: l'inflation et le chômage («l'inflation pousse l'électorat vers la droite, le chômage vers la gauche »). Si cette simplification peut être une base à partir de laquelle étendre le modèle (en intégrant d'autres variables, un intermédiaire médiatique dans la formation des opinions, etc.), l'absence totale d'effet des politiques décidées sur ces mêmes variables, y compris sous une forme extrêmement simplifiée, est par contre rédhibitoire et en change dramatiquement le sens. L'état de l'économie y est modélisé comme une succession de phases strictes de variations de l'inflation et du chômage, quels que soient les partis ou coalitions au pouvoir et les politiques qu'ils implémentent. Il est intéressant de penser que cette simulation se concentre sur les partis européens sur la période où les prérogatives nationales diminuent ( 1985-2005) et illustre finalement davantage l'impuissance politique des socio-démocrates, condamnés à courir après une économie sur laquelle ils ont renoncé à toute prise pour obtenir un pouvoir dont ils ne peuvent rien faire, qu'à une quelconque stratégie réellement politique.

Dans le même ordre d'idée, ou plutôt d'idéologie, un très grand nombre de simulations économiques font l'impasse complète sur l'existence de l'État et de son rôle économique (Taghawi-Nejad, 2013). Dans une sorte de far-west hayekien, les compagnies produisent, s'enrichissent, engagent et renvoient des travailleurs, sans interventions politiques, et on étudiera volontiers dans ce cadre le rôle de certaines contraintes "non-politiques » (l'exigence des employeurs - Takacs, Squazzoni, 2015, l'export - Catullo, 2013) sur ce « libre » marché " auto-régulé » et les stratégies des entreprises s'y ébattant. WorkSim (Goudet, 2013), au contraire, implémente l'État comme un agent du système et même si son rôle reste assez réduit, il dit quelque chose de fondamental sur les simulations économiques conçues comme discours. En effet, dans WorkSim, l'État est celui qui dit quels contrats de travail sont licites, donc quelles sont les interactions possibles entre les 
agents. C'est-à-dire que ce qui est habituellement un attribut du code (les agents ne peuvent effectivement faire que ce que leur code leur permet) est ici délégué à un agent interne à la simulation. En ce sens, cela nous permet de questionner la place silencieuse d'agents qui n'ont pas été codés comme tels, et qui pourtant déterminent les fondements du comportement des agents effectivement implémentés. Cette problématique nous amènera à interroger la place du simulateur dans sa simulation même, et trouve une source dans la manière dont il a conçu son modèle, c'est-à-dire des données et concepts dont il s'est emparé pour le construire.

\section{Calibrer}

Construire un modèle demande à être fondé empiriquement pour asseoir son propos. L'aspect le plus développé de ces fondements est celui de l'utilisation des données pour « calibrer » les modèles sur des savoirs empiriques. Cependant, ces données n'ont pu être produites et mobilisées que parce que ce qu'elles représentent a été préalablement conceptualisé par les simulateurs lorsqu'ils choisissent les données à partir desquelles ils travaillent.

\section{Quelles données?}

21 Le succès de la simulation des Anasazi a tenu en grande partie à son utilisation des données archéologiques (datation et localisation de la construction des habitations) et environnementales (climatologie, hydrologie et topologie de la vallée). S'agissant de reproduire fidèlement l'évolution des populations pour tenter d'en expliquer la disparition, la question de l'ajustement du modèle sur les données était en effet centrale. Mais des simulations extrêmement abstraites ne font pas moins usage de ce type de calibrage. Lynne Hamill et Nigel Gilbert proposent par exemple une méthode pour produire des réseaux de connexions entre individus plus proches des caractéristiques des réseaux de relations observables, sans en tirer d'application directe (Hamill, Gilbert, 2009). Cette étude fournit donc un outil pour la production de réseaux sociaux artificiels fondés empiriquement, qui sera utilisable dans des simulations qui ne concernent pas une situation précisément définie, mais gagnerait à travailler sur un réseau hypothétique plus réaliste.

Jusqu'ici, les processus de choix et de collecte des données ont été largement « informels : ni systématisés, ni documentés » (Edmonds, 2015). Pourtant, ces choix guident clairement la direction que prend une simulation et permet de déplacer sa discussion de l'adéquation modèle/données ("Est-ce que le modèle rend compte des données?») à celle des choix conceptuels qui ont guidé la collecte des données "pertinentes». Sous cet angle, on comprendra les appels à se saisir des immenses quantités de données générées par l'activité numérique de l'humanité comme une démission annoncée face à ce retour sur les concepts (Venturini et al., 2015). Se saisir de données massives parce qu'elles ont le mérite d'exister n'est qu'une réédition aux proportions titanesques de l'homme qui cherche ses clefs sous un lampadaire parce qu'il y a de la lumière. Et l'absence de questionnement sur leur mode de production, leur pertinence ou leur signification ne peut qu'annoncer des catastrophes intellectuelles. Contre cette tendance, une récente section spéciale du JASSS s'est attelée à explorer la possibilité d'amender le tropisme quantitatif encore actuel de la simulation. Sur un mode résolument auto-critique, Bruce 
Edmonds introduit cette section : «Les données qualitatives ont souvent été tenues pour 'non-scientifiques', critiquées parce que subjectives, biaisées, non-fiables et spécifiques au contexte [...] [ces] difficultés ne sont pas suffisantes pour justifier leur mise à l'écart » (Edmonds, 2015). Il est notamment question dans ce dossier d'introduire des méthodes d'enquête ethnologiques pour la construction d'un modèle, enquête toutefois orientée par un méta-modèle préalable, c'est-à-dire une articulation de concepts extrêmement génériques qui doivent être spécifiés pour chaque situation modélisée (Ghorbani et al., 2015). Ainsi, le travail conceptuel reste toujours en amont de la simulation comme des données qui la motivent, ce qui, étant donné la nature théorique de toute modélisation, est inévitable.

\section{Quels concepts?}

Bien que la constitution et l'articulation des concepts sociologiques soient au cœur du travail de formalisation des modèles de simulation, l'absence de leur discussion est un des points aveugles les plus saillants au sein du champ. Comme nous l'avons déjà souligné, leur questionnement est encore largement perçu comme "abstrus», et plus encore, manquant de "neutralité scientifique». L'histoire de la simulation au sein des sciences dures et de l'informatique n'est certainement pas étrangère à cette volonté de rester "scientifique ", " objectif ", et manque de voir qu'en devenant sociale, la simulation ne peut pas et, même, gagne scientifiquement à percevoir et affirmer ses inévitables partis pris.

24 Les simulations sont en effet porteuses d'un discours partisan dans leur conception même, bien en amont de l'interprétation de leurs résultats, et ces discours sont encore trop souvent sans fondements sérieux. Des travaux sur la diffusion d'opinions extrémistes en fournissent une illustration explicite (Deffuant, 2002). L'extrémisme y est d'abord conçu comme un concept abstrait, les opinions se distribuant sur une dimension simple allant de -1 à +1 . Ces travaux s'appuient sur une seconde variable, la certitude des agents quant à leurs propres opinions, les agents incertains étant plus susceptibles de changer d'opinion que les agents sûrs de leur fait, et tous ignorent les opinions trop divergentes de la leur : « Notre modèle est clairement lié à des observations psychologiques de bon sens [nous soulignons] : au-delà d'une certaine limite au désaccord, l'opinion des autres est considérée comme stupide et donc, ignorée; les gens sûrs d'eux tendent à être plus convaincants que ceux qui ne le sont pas » (Deffuant, 2003). On trouve régulièrement ce genre de justification par la trivialité du comportement des agents : «Peu nombreux sont ceux qui disputeraient le fait que nous évaluons notre "distinctivité" individuelle, [...] que nous agissons pour atteindre nos objectifs de distinction " (Smaldino, Epstein, 2015). Ou comme le précise encore Guillaume Deffuant, "des modèles populaires, comme les architectures d'agents "Belief Desire Intention" manipulent des concepts cognitifs de sens commun, peu fondés scientifiquement» (Deffuant, 2003). Cette architecture est l'une des plus utilisées, aux côtés de méthodes encore plus simples ${ }^{6}$ (Balke, Gilbert, 2014), et l'on peut en déduire qu'un très grand nombre de suppositions sur le comportement des agents sont pour ainsi dire faites au doigt mouillé. Cet état de fait est justifiable par les nécessaires simplifications de la modélisation, mais n'est pas moins justiciable des parti pris dans la mobilisation des concepts, et surtout, dans leur mise à l'écart par la stylisation. Une explication générative (le comportement des agents) peut sans doute rester très simple en première approche, mais il n'en faudra pas moins l'asseoir sur des 
concepts solides, y compris a posteriori, lorsque celle-ci a pu démontrer quelque pertinence pour expliquer le fait généré. Ici, comme pour les données, la documentation des choix est encore relativement faible et largement laissée à la justification par le fait (la simulation donne des résultats, donc les suppositions sont une bonne approximation).

\section{Quels paramètres ?}

La question partisane la moins discutée et pourtant sans doute la plus propre à la simulation est celle des éléments paramétrables des simulations. Cette question interroge non seulement la position du chercheur vis-à-vis de ce qu'il simule, mais exprime directement le rôle qu'il endosse par la position qu'il occupe au sein même de sa simulation au travers des paramètres qu'il manipule.

Une grande partie des simulations a une vocation explicative. Les paramètres y sont donc essentiellement un mode de production de variations permettant soit d'étudier différentes situations et de les comparer, soit de chercher les paramètres les plus adaptés à la reproduction générative d'une situation empirique. La position scientifique y est certainement surplombante mais il est communément admis que par leur complexité intrinsèque, «même les simulations les plus simples ne sont pas compréhensibles dans leur détail " (Edmonds, 2010). Ce caractère surplombant prend une tournure différente lorsqu'il est question de suggérer des politiques normatives: "Ironiquement, cette approche [la simulation] supposément "bottom-up" a mené à des politiques sociales "topdown" » (Venturini et al., 2015). Et l'on serait effectivement bien en peine de saisir la différence entre "prédire et contrôler », perçu comme trop "top-down", " hors-sol » et " comprendre et manager", ce qui serait plus "adapté à la complexité " (Squazzoni, 2014). Ou plutôt, on comprend une sophistication des outils du pouvoir que viendrait conseiller le simulateur, sans transformation politique aucune. La forme la plus simple de prise de recul sur l'identification à l'État est d'en faire un agent ordinaire du système, quoique doté de capacités différentes. En matière de régulation et de contrôle sanitaire de la chaîne de production alimentaire par exemple (Edmonds, 2010), l'État est l'instance qui décide du nombre de contrôleurs et de la stratégie de contrôle des établissements. Cette forme est en fait la forme plus directement utilisable à partir d'une compréhension partisane de la simulation : il s'agit pour un des agents du système d'établir une stratégie à partir de ses moyens dans un monde artificiel autonome, dont on a éventuellement par ailleurs pointé les manques conceptuels. Que cet agent soit ici l'État (ou du moins son incarnation locale) n'empêche pas d'imaginer des simulations partant d'un point de vue différent : « la formation de stratégie n'est plus réservée aux acteurs gouvernementaux » (Arhweiler, 2015).

Si l'instance décrétant le possible est paramétrable, comme c'est le cas dans WorkSim (Goudet et al., 2013), les agents sous son empire adaptent leurs comportements à ce qu'ils peuvent faire, c'est-à-dire, en l'occurrence, signer les types de contrats de travail disponibles. Cependant, il faut également considérer le fait que les agents peuvent "tricher ", c'est-à-dire briser le cadre prévu pour eux, ce qui n'est possible que si leur code leur permet de s'opposer à l'instance chargée cette fois de dire le légal, et non le strict possible. MAIA, un cadre de développement de simulation orienté sur le fonctionnement nécessairement institutionnel des faits sociaux propose cette possibilité (Ghorbani et al., 2013). Il prend pour exemple une simulation du retraitement informel des déchets informatiques en Inde et du travail des enfants qui, bien qu'interdit, est 
endémique dans ce secteur. Les agents y ont non seulement la possibilité de choisir d'obéir ou non aux normes édictées par les institutions (l'interdiction du travail des enfants), mais peuvent également réagir aux actes des agents chargés de faire respecter ces normes (ici, corrompre les inspecteurs du travail). Ces possibilités n'existent toutefois que pour les simulations où l'instance régulatrice est explicitement un agent. Comme nous l'avons déjà mentionné, si ces instances sont incorporées directement dans le comportement des agents, celles-ci se placent, en termes de discours tenu par la simulation, en dehors de la sphère du pensable politique : un marché artificiel sans cadre institutionnel place celui-ci, dont la présence est pourtant écrasante dans le comportement même des agents, hors de toute remise en question par le reparamétrage. De même, mais à front renversé, si les agents ont « désincorporé » la possibilité de sortir du cadre prévu, c'est-à-dire si leurs comportements sont conduits par des instances disciplinaires que l'on ne peut reparamétrer, la simulation comme discours place les comportements indisciplinés en dehors du pensable politique.

\section{Comparer}

On ne peut comprendre l'intérêt d'une simulation, en évaluer les forces et en critiquer les silences, qu'à l'aune d'un autre modèle dont on pointera en retour les manques et les inspirations. Cependant, de même que l'explicitation du choix des données, la présentation des modèles, de leur fonctionnement, des outils utilisés, de leurs résultats est une jungle de pratiques hétérogènes à tous les niveaux.

\section{« L'anarchie règne »}

Malgré les avertissements et appels répétés à une forme de discipline des chercheurs du domaine, le modèle ad hoc est la règle dans la simulation sociale. Si cette question était déjà discutée lorsque le champ naissait à peine (Woolridge, Jennings, 1998), les constats ultérieurs (Richiardi et al., 2006 ; Müller et al., 2013 ; Collins et al., 2015) rendent compte, avec alarmisme, de l'éclatement toujours actuel des méthodes, outils et normes de présentation du modèle et de ses résultats, qui empêche la comparaison de modèles à modèles, voire interdise la réplicabilité de leurs résultats (Rouchier et al., 2008).

Comme diverses méta-analyses (Heath et al., 2009) (Thiele et al., 2012) le montrent, les langages préférés des simulateurs ne sont pas des langages spécialisés en simulation sociale, mais sont des langages de programmation génériques plutôt orientés-objet $(\mathrm{C} / \mathrm{C}+$ +/objective-C et Java, principalement), soit des plates-formes multi-agents génériques, mais pas spécialement dédiées à la simulation sociale (Netlogo et Repast, essentiellement). De plus, aux côtés des nombreux langages minoritaires, un nombre important de simulations ne précisent même pas les outils de modélisation utilisés, ajoutant une couche d'opacité à cette précaire tour de Babel. L'UML (Unified Modeling Language), alors qu'il est très largement reconnu en informatique comme un standard de communication indépendant des langages de programmation et qu'il a été étendu depuis longtemps pour la représentation des agents (Odell et al., 2000), reste assez peu utilisé (Bersini, 2012). D'autres appels à construire des normes de présentation des simulations, comme le protocole ODD (Overview, Design concepts, Details; Richiardi et al., 2006) et son extension ODD + D (ODD + Decision; Müller et al., 2013), sont également suivis d'effets très 
relatifs et servent davantage de vague guide de pratiques souhaitables que de normes contraignantes que les éditeurs de journaux auraient à charge de faire respecter.

31 Un avenir possible de la simulation sociale serait ainsi un succès paradoxal comme méthode, mais sa disparition comme champ de recherche autonome qui, faute d'un langage commun, aurait éclaté en fragments thématiques. Ses problématiques seraient donc entièrement déterminées par les champs dans lesquels elle est utilisée, laissant sa critique être menée fragmentairement au sein des champs qui s'en sont emparés. Il faudrait pourtant en partie prendre cette crainte à contre-pied. Si le manque de lisibilité des modèles et de leurs résultats est un problème en soi (qui menace bien plus que l'unité du champ), la multiplication des simulations, méthodes d'interprétation, et outils de conceptualisations ad hoc permet un foisonnement expérimental dont la souplesse rapproche (ou, du moins, pourrait rapprocher) des disciplines et thématiques autrement difficiles à faire cohabiter. Cependant, en l'absence de questions transversales de fond, le champ de la simulation est condamné à n'être que le lieu où les questions d'ordre technique se posent, mais dont nul sens ne saurait être produit en commun: Que permettent les plates-formes disponibles? (Kravari , Bassiliades, 2015) Quelles méthodes d'extraction des résultats d'une simulation? (Lee et al., 2015) C'est-à-dire qu'il faudrait moins s'inquiéter du manque de normes, qui n'affecte en rien l'hétérogénéité des simulations, que de l'absence de cadres critiques solides permettant d'analyser, de dire le sens, pointer les manques, dévoiler les orientations, et établir des rapports entre des modèles et des résultats dont la diversité est la source même des cadres critiques, précisément parce qu'ils sont tous issus d'une même technique dont on comprend les rouages conceptuels communs.

\section{La critique comme méta-modèle}

La critique d'une simulation doit s'attaquer avant tout à ses structures formelles. Que l'on imagine d'autres facteurs explicatifs, d'autres comportements possibles mettant à mal son fonctionnement, voire que l'on objecte aux conclusions dont celle-ci propose une explication générative, ces critiques se fondent nécessairement sur un contre-modèle, formalisé ou non. Construire un cadre critique réclame un niveau d'abstraction supplémentaire: un méta-modèle suffisamment abstrait pour être (contre-)«toutterrain ", mais suffisamment spécifié pour n'être pas qu'une forme de contrôle technique, bien trop peu exigeante du point de vue de la critique sociale.

Les modèles globaux peuvent être compris comme une tentative de produire un cadre peu abstrait mais aux structures complexes, contre lequel peut être éprouvé un modèle particulier, qui doit parvenir à des conclusions locales acceptables pour le système global. Ces modèles sont nés de la généralisation de problèmes " locaux ", industriels (Forrester, 1961), urbains (Forrester, 1969), nationaux ${ }^{7}$, avant d'être appliqués au monde compris comme système (Meadows et al., 1972). Ce mode de construction d'un cadre critique présente un défaut important : ces méga-modèles ne sont pas eux-mêmes au-delà de la critique, qui doit s'en prendre à leurs concepts. À nouveau, la critique de la simulation ne peut se placer que sur son terrain d'expression: l'espace théorique. Cela n'est pas sans poser problème puisque, au contraire de ce que l'on en attend spontanément, cet espace de la théorie est avant tout traité par la simulation comme un espace expérimental, pour lequel l'explication générative est finalement une modalité méthodologique qui « garde la théorie sous contrôle» (Healy, 2015), un moyen de juger les théories expérimentales. 
L'approche inverse, qui est de fait la norme de la simulation sociale, n'est pas moins problématique : un cadre théorique trop peu exigeant, strictement limité à des aspects techniques implémentés comme langage multi-agents (agents autonomes, hétérogènes, situés, etc.) qui ne disent rien de ce qu'est un fait social, et ne permettent donc de disqualifier des simulations sociales que sur des critères liés au fait simulationnel, et pas du tout au fait social. Si cette trop large ouverture a, au moins partiellement, permis la rencontre disciplinaire sur un terrain commun, celui-ci reste de ce fait sans consistance propre. Nous conjecturons que ce qui lui donnera réellement consistance dans les sciences humaines et sociales (une polarisation forte en différents partis pris qui ne se résument pas à une division en discipline préexistante), sera vécu comme déchirement par ce champ encore fortement marqué par des ambitions de neutralité scientifique issues des sciences dures. Cette structuration des oppositions, si elle n'éclate pas le champ, se présentera nécessairement sous la forme de méta-modèles concurrents, répondant à des questions d'une très grande généricité (Qu'est-ce qu'un fait social? De quoi est-il nécessairement composé ? Comment ces éléments peuvent-ils être structurés ? ), qui ensuite guident l'interprétation comparative partisane des discours tenus par les simulations.

\section{Comparer les discours}

34 La constitution de ces méta-modèles passe par la reconstruction des concepts communs des simulations, c'est-à-dire structurer l'analyse des discours qu'ils tiennent sur des objets hétérogènes, à partir de concepts communs. La puissance de l'«anarchie régnante » est ici importante puisque c'est par elle qu'il est possible de faire circuler des conclusions les unes contre les autres. Précisons qu'il n'est pas ici question de se cantonner à la comparaison de simulations proches traitant sur le même mode des mêmes objets, avec des variations somme toute mineures. Il s'agit de se saisir des concepts mobilisés de façons diverses pour en confronter les usages.

Si l'on prend l'exemple récurrent de l'identité, celle-ci tient des fonctions très différentes selon les simulations, mais le concept général nous permet de tenir ensemble leurs conclusions :

- La simulation de ségrégation de Schelling l'utilise pour mesurer ce que la ségrégation doit à la volonté de non-mixité. (Schelling, 1971);

- Une explication générative a été proposée pour la domination de l'ethnocentrisme dans des jeux de coopération/trahison sur des bases strictement identitaires. (Hammond, Axelrod, 2006 ; Hartshorn, 2013) ;

- Cependant, sans en remettre en question que la domination évolutive des stratégies identitaires, une de ses extensions démontre que si ces identités peuvent être apprises et construites par les agents, les identités culturelles dominent nettement les stratégies identitaires brutes (Hales, Edmonds, 2015);

- Les simulations sur la circulation d'opinions extrémistes montrent comment la stabilité de l'identité culturelle (l'opinion) attire une très grande masse d'agents incertains, et plus en détail, comment des micro-groupes aux identités fortes, proches mais étanches se retrouvent soudainement liés et dépassés par la masse d'incertains attirés dans leur direction générale (Deffuant, $2002 ; 2003$ );

- Une simulation de l'effet identitaire dans les mécaniques de la protestation de travailleurs autochtones et immigrés montre comment la défiance identitaire entre travailleurs conduit 
à des protestations faibles, localisées et peu durables, dont la répression n'a aucun mal à venir à bout (Kim, Hanneman, 2011). populations hétérogènes ne peut se produire que si les identités essentialisées ne sont pas sources de ségrégation interne. La construction d'une identité culturelle commune s'annonce comme source de puissance meilleure que les stratégies étroitement ethnicistes, et peut naître d'un ensemble de groupes sûrs de leur fait, proches mais encore séparés, qui seront dépassés par la masse circulante des protestataires. Bien entendu, cette synthèse reste simpliste, et sa base uniconceptuelle n'y est pas étrangère. Nous voulions simplement montrer comment un concept ici encore pré-théorique sert déjà de pont entre des simulations et peut articuler les simulations entre elles, donc préfigurer un méta-modèle, cette fois structure multi-conceptuelle, qui servirait de grille formelle de lecture des simulations.

\section{(Ré)Interpréter}

Toute interprétation d'une simulation serait ainsi effectuée à travers une grille de lecture formalisée. Et si cette grille de lecture considère les spécificités de la simulation, y compris dans ses fondements impensés, celle-ci rend possible le dialogue critique avec et entre les discours tenus sur et par ces simulations.

\section{Interpréter les résultats}

L'un des résultats abstraits des simulations sur l'extrémisme (Deffuant, 2002) est que si les extrémistes sont sûrs de leur fait et le centre ne l'est pas, une des positions extrêmes attirera la grande majorité du centre. La nature des opinions considérées n'étant pas spécifiée, il conviendrait de considérer que, selon les cas, la position partisane de l'interprète est différente et "l'extrémisme" devient ou non désirable. Ainsi, la simulation générique "ne devrait pas être interprétée normativement» (Weisbuch, 2015). Sans prendre ce genre de précautions, une lecture en conclut, à propos de la montée d'«attitudes anti-minorités» en Allemagne, qu'il faut s'inquiéter «du fait que tant d'Allemands sont incertains de leurs opinions» (Von Randow, 2003). Dans ce cadre d'application, on pourrait très logiquement s'inquiéter davantage du fait que l'extrême opposé n'ait pas le poids nécessaire à emporter l'opinion allemande dans son ensemble. On voit ici comment différentes lectures partisanes des résultats théoriques d'une simulation conduisent à différents diagnostics. Il faut noter que ces interprétations sont le fait de grilles de lecture extérieures à la logique de la simulation elle-même, c'est-à-dire que l'interprétation des résultats, même revêtue de scientificité, appartient nécessairement à la circulation ordinaire des opinions politiques. Cependant, il existe une part d'erreur factuelle grave lorsque certaines limites du fait simulatoire même ne sont pas prises en considération.

La simulation de ségrégation de Schelling conduit à la conclusion qu'il suffit que chaque agent veuille assez peu de voisins similaires pour produire des sociétés spatialement ségréguées. Il faut tout de même amender dramatiquement cette conclusion. Il est vrai que le taux d'auto-similarité moyen entre voisins est toujours supérieur au minimum désiré par les agents. Mais il faut noter qu'une répartition aléatoire des agents produit un taux moyen de similarités entre voisins de $50 \%$, qui est donc un plancher indépassable 
même en l'absence de tout désir de non-mixité. Par ailleurs, un désir de non-mixité plus fort conduit très logiquement à une non-mixité plus forte (visible à l'œil nu sur l'expression graphique de la simulation, mais également mesurable statistiquement), à l'exception des grilles non-convergentes. Celles-ci se produisent lorsque le désir de nonmixité est tellement fort (autour de $80 \%$ en fonction de la densité de population) que l'écrasante majorité des agents sont insatisfaits en permanence, et chaque mouvement ne fait qu'annoncer le suivant. Or, on lit encore aujourd'hui sous la plume de chercheurs: "Cela suggère que, globalement, les quartiers mixtes seraient composés de méchants intolérants essayant d'en échapper, et que les quartiers ségrégués seraient composés de sympathiques personnes tolérantes gênées de vivre là, mais pas assez pour en partir. Nous ne sommes pas sociologues, mais nous suspectons que la recherche confirmerait ces attentes" (Thomson, Berr, 2009). Et ce, alors même que le modèle ne converge pas et produit donc une société de racistes en insatisfaction-migration permanente. Nous ne sommes pas historiens, mais nous suspectons qu'un examen même superficiel de l'histoire humaine confirmerait que des sociétés d'identitaires durablement mécontents de leur voisinage ne produisent pas une société mixte, mais lynchages, massacres et génocides. «Nous espérons que [Thomson et Berr] retireront leurs remarques [...] Nous craignons que, dans un futur proche, les étudiants de nos classes commencent à défendre ces idées » (Thomson, Berr, 2009).

Trêve d'ironie: pour une société d'individus refusant la proximité d'une altérité, la possibilité de passer à la violence plutôt qu'au déménagement tient d'un réalisme presque naï, mais trop bien fondé pour être ignoré. Une population qui passe sa vie à être mécontente et à fuir n'est pas une société mixte mais une société en pleine implosion. Cette possibilité irréductible du passage à la violence de tout conflit est à la fois inscrite et lisible dans la simulation même, ici par l'insatisfaction permanente qui l'empêche de converger, mais se trouve reléguée à sa marge extrême parce qu'elle n'est pas implémentée comme telle. Si cette implémentation n'est pas nécessaire, il convient de mentionner que la bonne lecture d'une simulation demande de garder en tête la possibilité de l'explosion du cadre, dont le comportement du système en donne les indices sans que le code en fasse mention.

\section{Interpréter le code}

Comme nous l'avons déjà fait remarquer, lorsqu'une simulation limite les possibilités de ses agents, il faut y lire non pas un manque de la simulation mais la présence invisible d'institutions de régulation dans la simulation même. En conséquence, interpréter le discours tenu autour d'une simulation, c'est d'abord interpréter son code, ou du moins son expression formelle (en pseudo-code, équations, schémas UML, etc.). Plus exactement peut-être, il s'agit de considérer le code de la simulation et d'en extraire ce que sa dynamique peut dire.

En effet, le code étant lui-même une abstraction parfaitement formelle, l'interprétation doit tenir compte de cette caractéristique pour en tirer les sens possibles. Les simulations d'opinions extrémistes sont à ce titre explicitement réinterprétables : politiquement, des «droits civiques » à des situations plus «dramatiques », ou des opinions sur «l'art et la mode ", « la consommation de produit de luxe », etc. (Weisbuch, 2015) Cependant, il est possible de réinterpréter une simulation qui s'y prête apparemment moins, mais dont le code est tout aussi abstrait et le permet donc tout aussi bien. Si la simulation de 
ségrégation de Schelling est considérée sous un angle politique par exemple, il est possible de l'observer comme une stylisation des dynamiques de "ségrégation » entre courants d'un même parti par exemple. Une illustration de ce que le degré de sectarisme politique individuel produit au niveau du parti et conduit à la même limite que précédemment : la possibilité d'un parti de sectarismes permanents, jusqu'à l'instable, la violence ou du moins, l'éclatement du cadre dans lequel les agents interagissent, la scission. De la même façon, on pourrait y lire les partitions au sein de l'université : la volonté de travailler principalement entre-soi (quelles que soient la définition et l'échelle de cet entre-soi) produit au niveau de l'université globale un très fort clivage entre les courants, jusqu'au point de scission si d'aventure il était question de ne quasiment jamais avoir à faire aux "autres». La récente tentative de fonder une section alternative d'économie est sans doute une illustration assez nette de cette possibilité. Mais plus en profondeur, et parce que le code est toujours aussi abstrait, on peut en renverser les termes. Dans l'esprit original, l'identité est attachée aux agents et ceux-ci peuvent se déplacer entre des localisations identifiables à des ressources (toutes équivalentes dans la simulation originale). On peut considérer l'identité comme modifiable (comme une opinion, par exemple), et spatialisée au sens le plus mathématique du terme : l'espace des opinions est ici bidimensionnel. Par ailleurs, les ressources peuvent être pensées comme globalement attachées à l'agent, par sa trajectoire sociale générale. On peut donc tirer de la même simulation que plus les agents veulent rester dans un entre-soi économique, plus les identités/opinions auront tendance à être clivées et ségréguées entre les deux catégories économiques, éventuellement jusqu'au point d'explosion sociale. C'est-à-dire que d'une simulation cherchant à montrer que la volonté d'entre-soi identitaire produit une ségrégation dans l'accès aux ressources (spatialisées), on peut faire dire que la volonté d'entre-soi économique produit une ségrégation dans les positions culturelles (spatialisées). Cela n'est possible que parce que l'abstraction du code de la simulation en fait une incarnation du principe d'équivalence structurale. La simulation proprement dite ne saisit pas l'équivalent structural, les liens structurels entre deux situations concrètes, elle est l'équivalence structurale à un degré tel qu'elle est l'expression du lien lui-même. Il s'agit donc pour l'interprétation critique de la simulation sociale, comprise comme systématique d'exploration théorique, d'établir de quoi la simulation est l'équivalent.

Si l'on ne se fie qu'aux apparences, l'approche « bottom-up » de la simulation sociale peut être associée à une forme de l'individualisme méthodologique. Celle-ci semble en effet partir des actions individuelles pour en déduire des conséquences émergentes à l'échelle de la société, conçue comme simple collection d'individus en interactions. Nous ne sommes pas les seuls à penser que c'est un tort (Marchionni, Ylikoski, 2013), mais nous voulons ajouter ici que la simulation est, par ses principes mêmes, une expression du structuralisme.

Dans une simulation, les agents ne peuvent agir que dans la stricte mesure où les structures de la simulation le leur permettent, les structures les déterminent donc intégralement. Plus précisément, leurs comportements encodés sont intégralement dus à des structures qui n'apparaissent pas comme telles mais n'en sont pas moins : le système de la simulation dans laquelle ils agissent définit strictement ce qu'il leur est possible de percevoir, ce qu'il leur est possible de faire, jusqu'à la façon dont leurs processus de 
cognitions mènent des premières aux secondes. Le code des agents peut paraître individualisé par l'autonomie qui leur est accordée, mais il faut noter que d'une manière très générale, tous les agents se comportent selon le même code. L'algorithme comportemental, s'il permet largement des comportements différenciés, n'en dit pas moins que les agents sont conduits par les stricts mêmes déterminants. Or, un déterminant intégralement partagé est-il autre chose que le produit d'une structure de régulation sociale? Que celle-ci ne soit pas exprimée explicitement en tant que structure dans le code rend sa présence d'autant plus implacable qu'elle y est en quelque sorte naturalisée par le code même des agents « individuels » : les agents se comporteraient de telle façon parce que «c'est ainsi » et pas parce qu'une détermination extérieure les y pousserait. Le fait que le simulateur soit la main qui introduit ces déterminations n'est encore que superficiel, et le rappeler n'enfoncerait que des portes ouvertes. Il est entendu que le simulateur ne peut qu'introduire ses propres conceptions, mais ce qu'il introduit ne correspond pas nécessairement à ses intentions. C'est que les comportements les plus « naturels " dans le système artificiel sont en fait l'expression stylisée de structures sociales déterminantes, toujours présentes au sein même des simulations bien que globalement impensées.

La perception, par exemple, est conçue sur le mode de l'évidence: il y a des objets à percevoir, ceux-ci sont donc perçus. Mais on peut penser deux propriétés importantes qui mettent à mal cette lecture. Tout d'abord, la perception à ce degré de stylisation représente une perception médiée par un ensemble de structures non-dites. Les agents de Schelling perçoivent l'identité de leur voisinage, mais ils le perçoivent nécessairement par le biais d'un langage ou de technologies médiatiques, donc des structures immédiatement sociales, qui permettent la production de ces identités différenciées. Et c'est là le second élément : les objets n'ont des propriétés encodées que parce que celles-ci sont perceptibles. La structure invisible est à la fois dans le code comportemental de l'agent et dans celui de ce qu'il peut " voir », qui ne peuvent faire sens l'un sans l'autre. Il en va de même pour toutes les actions des agents qui sont médiées par toutes sortes d'outils et techniques sociales non-dites (un moyen de transport, un marché, etc.), qui construisent les objets potentiels sur lesquels s'exercent les actions des agents. Ce n'est pas parce que des objets sont là que les agents ont prise sur eux, mais simultanément, parce que les agents sont outillés pour les transformer/détruire/subir que ces objets et propriétés sont là. Et il en va encore de même pour les processus de cognition : les agents ne peuvent raisonner et désirer qu'à propos d'objets et propriétés qui leur sont intelligibles ou sur lesquels ils ont prise. Ainsi, les objets et agents n'ont de sens que les uns par rapport aux autres au sein du système qui les lie. On ne peut pas « déplacer » un agent d'une simulation dans une autre parce qu'il lui manquerait l'outillage de perception et d'action capable de faire sens dans cette nouvelle simulation, parce que celui-ci fait entièrement système avec le modèle dont il est partie prenante.

Il est donc finalement question, pour la critique des simulations, d'en « déplier » le code, d'en mettre au jour les naturalismes pseudo-individualisés, et y lire les institutions et structures qui leur permettent de faire système. Et par le biais bien compris de cet outil commun, le "dialogue interdisciplinaire » pourrait ainsi être réinvesti par les savoirs critiques: si des physiciens aspirent à faire sociologie, des sociologues peuvent «faire physique ». 


\section{BIBLIOGRAPHIE}

American review of sociology 110 (2005)

Arhweiler (Petra), Schilperoord (Michel), Pyka (Andreas), Gilbert (Nigel), « Modelling research policy : ex-ante evaluation of complex policy instruments ", Journal of Artificial Societies and Social Simulation, vol. 18, $\mathrm{n}^{\circ} 4,2015$, http://jasss.soc.surrey.ac.uk/18/4/5.html

Angus (Simon), Hassani-Mahmooei (Behrooz), « "Anarchy” reigns: a quantitative analysis of agent-based modelling publication practices in JASSS, 2001-2012 ", Journal of Artificial Societies and Social Simulation, vol. 18, $\mathrm{n}^{\circ} 4$, 2015, http://jasss.soc.surrey.ac.uk/18/4/16.html

Axelrod (Robert), The evolution of cooperation, New York, Basic Books, 1984.

Baker (Alan), « Simulation-based definitions of emergence », Journal of Artificial Societies and Social Simulation, vol. 13, $\mathrm{n}^{\circ}$ 1, 2010, http://jasss.soc.surrey.ac.uk/13/1/9.html

Balke (Tina), Gilbert (Nigel), « How do agents make decisions ? A survey », Journal of Artificial Societies and Social Simulation, vol. 17, $\mathrm{n}^{\circ}$ 4, 2014, http://jasss.soc.surrey.ac.uk/17/4/13.html

Benard (Stephen), Willer (Robb), « A wealth and status-based model of residential segregation », Journal of mathematical sociology, vol. 31, $\mathrm{n}^{\circ} 2$, pp 49-74, 2007

Bersini (Hugues), « UML for AMB », Journal of Artificial Societies and Social Simulation, vol. 15, $\mathrm{n}^{\circ} 1$, 2012, http://jasss.soc.surrey.ac.uk/15/1/9.html

Castro (Rodrigo), Jacovkis (Pablo) « Computer-based global models : from early experiments to complex systems », Journal of Artificial Societies and Social Simulation, vol. 18, n 1, 2015, http:// jasss.soc.surrey.ac.uk/18/1/13.html

Catullo (Ermanno), « An agent based model of monopolistic competition in international trade with emerging firm heterogeneity ", Journal of Artificial Societies and Social Simulation, vol. 16, $\mathrm{n}^{\circ} 2$, 2013, http://jasss.soc.surrey.ac.uk/16/2/7.html

Cecconi (Frederico), Campenni (Marco), Andrighetto (Giulia), Conte (Rosaria) « What do agentbased and equation-based modelling tell us about social conventions: the clash between ABM and EBM in a congestion game framework ", Journal of artificial societies and social simulation, vol. 13, $\mathrm{n}^{\circ}$ 1, 2010, http://jasss.soc.surrey.ac.uk/13/1/6.html

Centola (Damon), Wilenski (Uri), McEnzie (Eamon), « A hands-on modeling approach to evolution: learning about the evolution of cooperation and altruism through multi-agent modeling - the EACH project », International Conference of the Learning Science, 2000

Collins (Andrew), Petty (Mikel), Vernon-Bido (Daniele), Solomon (Sherfey), « A call to arms : standards for agent-based modeling and simulation ", Journal of Artificial Societies and Social Simulation, vol. 18, $\mathrm{n}^{\circ}$ 3, 2015, http://jasss.soc.surrey.ac.uk/18/3/12.html

Conte (Rosaria), Gilbert (Nigel), Bonelli (Giulia), Cioffi-Revilla (Claudio), Deffuant (Guillaume), Kertesz (Janos), Loreto (Vittorio), Moat (Suzy), Nadal (Jean-Pierre), Sanchez (Angel), Nowak (Andrzej), Flanche (Andreas), San Miguel (Maxi), Helbing (Dirk), « Manifesto of computational social science », The European pysical Journal. Special Topics, vol. 214, n 1, pp. 325-346, 2012

Dean (Jeffrey), Gummerman (George), Epstein (Joshua), Axtell (Robert), Swedlund (Alan), Parker (Miles), Mccaroll (Steven), « Understanding Anasazi culture change through agent-based 
modeling ", Dynamics in human and primate societies : agent-based modeling of social and spatial process , Santa Fe institute, New York \& London : Oxford university press, 2000

Deffuant (Guillaume), Amblart (Frédéric), Weisbuch (Gérard), Faure (Thierry), « How can extremism prevail? A study based on the relative agreement interaction model », Journal of Artificial Societies and Social Simulation, vol. 5, $\mathrm{n}^{\circ}$ 4, 2002, http://jasss.soc.surrey.ac.uk/5/4/1.html Deffuant (Guillaume), Weisbuch (Gérard), Amblart (Frédéric), Faure (Thierry), « Simple is beautiful... and necessary ", Journal of Artificial Societies and Social Simulation, vol. 6, n 1, 2003, http://jasss.soc.surrey.ac.uk/6/1/6.html

Deffuant (Guillaume), Moss (Scott), Jager (Wander), « Dialogues concerning a (possibly) new science ", Journal of Artificial Societies and Social Simulation, vol. 9, n 1, 2006, http:// jasss.soc.surrey.ac.uk/9/1/1.html Drogoul (Alexis), De la simulation multi-agent à la résolution collective de problèmes, une étude de l'émergence de structures d'organisation dans les systèmes multi-agents, Thèse de doctorat à l'université paris 6, 1993.

Edmonds (Bruce), « Bootstrapping knowledge about social phenomena using simulation models ", Journal of Artificial Societies and Social Simulation, vol. 13, n 1, 2010, http:// jasss.soc.surrey.ac.uk/13/1/8.html

Edmonds (Bruce), « Using qualitative evidence to inform the specification of agent-based models ", Journal of Artificial Societies and Social Simulation, vol. 18, n 1, 2015, http:// jasss.soc.surrey.ac.uk/18/1/18.html

Epstein (Joshua), « Agent-based computational models and generative social sciences », Complexity, vol. 4, n 5, pp. 41-60, 1999

Epstein (Joshua), Generative social science, Princeton university press, 2006

Epstein (Joshua), « Why model ? ", Journal of Artificial Societies and Social Simulation, vol. 11, $\mathrm{n}^{\circ} 4$, 2008, http://jasss.soc.surrey.ac.uk/11/4/12.html

Forrester (Jay), Industrial systems, Waltham, MA : Pegasus communications, 1961

Forrester (Jay), Urban dynamics, Waltham, MA : Pegasus communications, 1969

Galam (Serge), Sociophysics : a physicist's modeling of psycho-political phenomenas, New York, Springer-Verlag, 2012

Gardner (Martin), « Mathematical Games. The fantastic combinations of John Conway's new solitaire game « life » ", Scientific American, n² 223, pp. 120-123, 1970

Ghorbani (Amineh), Bots (Pieter), Dignum (Virginia), Dijkema (Gerard), « MAIA: a framework for developing agent-based social simulations ", Journal of Artificial Societies and Social Simulation, vol. 16, $\mathrm{n}^{\circ}$ 2, 2013, http://jasss.soc.surrey.ac.uk/16/2/9.html

Ghorbani (Amineh), Dijkema (Gerard), Schrauwen (Noortje), « Structuring qualitative data for agent-based modeling ", Journal of Artificial Societies and Social Simulation, vol. 18, n 1, 2015 http:// jasss.soc.surrey.ac.uk/

Gilbert (Nigel), Den Besten (Matthijs), Bontoviks (Akos), Craenen (Bart), Divina (Frederico), Eiben (Gusz), Griffioen (Robert), Hévìzi (György), Lõrincz (Andras), Schuster (Stephan), Schut (Martin), Tzolov (Christian), Vogt (Paul), Yang (Lu), « Emerging artificial societies through learning », Journal of Artificial Societies and Social Simulation, vol. 9, n², 2006 http://jasss.soc.surrey.ac.uk/ 
Goudet (Olivier), Kant (Jean-Daniel), Ballot (Gérard), « A multi-agent model of the french labor market : WorkSim » (2013), 18th Annual Workshop on Economic Science with Heterogeneous Interacting Agents, Reykjavik university, 2013.

Granjon (Fabien), « Du pragmatisme et des technologies numériques », Hermès, n 73, 2015, pp. 215-220.

Grimm (Volker), Berger (Uta), Bastiansen (Finn), Eliassen (Sigrunn), Ginot (Vincent), Giske (Jarl), Goss-Custard (John), Grand (Tamara), Heinz (Simone), Huse (Geir), Huth (Andreas), Jepsen (Jane), Jørgensen (Christian), Mooij (Wolf), Müller (Birgit), Pe'er (Guy), Piou (Cyril), Railsback (Steven), Robbins (Andrew), Robbins (Martha), Rossmanith (Eva), Strand (Espen), Souissi (Sami), Stillman (Richard), Vabø (Rune), Visser (Ute), DeAngelis (Donald), « A standard protocol for describing individual-based and agent-based models », Ecological Modelling vol. 198, n 1-2, pp. 115-126, 2006

Hales (David), Edmonds (Bruce), « Culture trumps ethnicity !- Intra-generational cultural evolution and ethnocentrism in an artificial society », Center for Policy Modeling, Manchester metropolitan university, 2015

Hamill (Lynne), Gilbert (Nigel), « Social circles », Journal of Artificial Societies and Social Simulation, vol. 12, n 2, 2009, http://jasss.soc.surrey.ac.uk/12/2/3.html

Hammond (Ross), Axelrod (Robert), « The evolution of ethnocentrism », Journal of conflict resolution, $\mathrm{n}^{\circ}$ 50, pp. 926-936, 2006

Hartshorn (Max), Kaznatcheev (Artem), Schultz (Thomas), « The evolutionary dominance of etchnocentric cooperation ", Journal of Artificial Societies and Social Simulation, vol. 16, $\mathrm{n}^{\circ} 3,2013$, http://jasss.soc.surrey.ac.uk/16/3/7.html

Hatna (Erez), Benenson (Itzhak), « The Schelling model of ethnic residential dynamics : beyond the integrated-segregated dichotomy of patterns ", Journal of Artificial Societies and Social Simulation, vol. 15, $\mathrm{n}^{\circ}$ 1, 2012, http://jasss.soc.surrey.ac.uk/15/1/6.html

Healy (Kieran) « Fuck Nuance », American Sociological Association Meeting, Chicago, 2015, http://kieranhealy.org/files/papers/fuck-nuance.pdf

Heath (Brian), Hill (Raymond), Ciarallo (Franck), « A survey of agent-based modeling practices (January 1998 to July 2008) », Journal of Artificial Societies and Social Simulation, vol. 12, $\mathrm{n}^{\circ}$ 4, 2009, http://jasss.soc.surrey.ac.uk/12/4/9.html

Kim (Jae-Woo), Hanneman (Robert), « A computational model of worker protest », Journal of Artificial Societies and Social Simulation, vol. 14, n 3, 2011 http://jasss.soc.surrey.ac.uk/14/3/1.html Kravari (Kalliopi), Bassiliades (Nick), « A survey of agent platforms », Journal of Artificial Societies and Social Simulation, vol. 18, n 1, 2015, http://jasss.soc.surrey.ac.uk/18/1/11.html

Lee (Ju-Sung), Filatova (Tatiana), Liegmann-Zilinska (Ariga), Hassani-Mahmooei (Behrooz), Stonedahl (Forrest), Lorscheid (Iris), Voinov (Alexey), Polhill (Gary), Sun (Zhanli), Parker (Dawn), "The complexities of agent-based modeling output analysis ", Journal of Artificial Societies and Social Simulation, vol. 18, $\mathrm{n}^{\circ} 4,2015$, http://jasss.soc.surrey.ac.uk/18/4/4.html

Macy (Michael), Flache (Andreas), « Social Dynamics from the bottom-up : Agent-based models of social interaction », in Hedström (Peter) \& Bearman (Peter), The oxford handbook of analytical sociology, chapitre 11, Oxford university press.

Manzo (Gianluca) (dir.), « La simulation multi-agents : principes et applications aux phénomènes sociaux », Revue française de sociologie, vol. $55, \mathrm{n}^{\circ} 4,2014$ 
Marchionni (Caterina), Ylikoski (Petri), « Generative explanation and individualism in agentbased simulation », Philosophy of the social sciences, vol. 43, n 3, pp. 323-340, 2013

Meadows (Donnela), Meadows (Dennis), Randers (Jørgen), Behrens (William), The limits to growth, New York, Universal books, 1972

Moss (Scott), Edmonds (Bruce), « Toward good social sciences », Journal of Artificial Societies and Social Simulation, vol. 8, $\mathrm{n}^{\circ}$ 4, 2005, http://jasss.soc.surrey.ac.uk/8/4/13.html

Müller (Birgit), Bohn (Friedrich), Dressler (Gunar), Groeneweld (Jürgen), Klassert (Christian), Martin (Romina), Schlüter (Maja), Schulze (Jule), Weisse (Hanna), Schwarz (Nina), « Describing human decisions in agent-based models - ODD + D, an extension of the ODD protocol », Environmental modeling \& software $\mathrm{n}^{\circ} 48, \mathrm{pp} .37-48,2013$

Odell (James), Van Dyke Parunak (H.), Bauer (Bernhard), « Extending UML for Agents », Proceedings of the Agent-Oriented Information Systems Workshop at the 17th National conference on Artificial Intelligence, Austin, Texas, 2000

Orléan André (dir.), À quoi servent les économistes s'ils disent tous la même chose : manifeste pour une économie pluraliste, Paris, Les liens qui libèrent, 2015

Proceedings of the national academy of science 99 (3) (2002)

Richiardi (Matteo), Leombruni (Roberto), Saam (Nicole), Sonessa (Michele), « A common protocol for agent-based social simulations ", Journal of Artificial Societies and Social Simulation, vol. 9, $\mathrm{n}^{\circ} 1$, 2006, http://jasss.soc.surrey.ac.uk/9/1/15.html

Rouchier (Juliette), Cioffi-Revilla (Claudio), Polhill (Gary), Takadama (Keiki), « Progress in modelto-model analysis ", Journal of Artificial Societies and Social Simulation, vol. 11, n² 2, 2008, http:// jasss.soc.surrey.ac.uk/11/2/8.html

Rossiter (Stuart), Noble (Jason), Bell (Keith), « Social Simulations: Improving Interdisciplinary Understanding of Scientific Positioning and Validity », Journal of Artificial Societies and Social Simulation, vol. 13, $\mathrm{n}^{\circ}$ 1, 2010, http://jasss.soc.surrey.ac.uk/13/1/10.html

Schelling (Thomas), « Dynamic Models of Segregation », Journal of Mathematical Sociology, $\mathrm{n}^{\circ} 1$, pp.143-186, 1971

Seltzer (Nicholas), Smirnov (Oleg), « Degrees of Separation, Social Learning, and the Evolution of Cooperation in a Small-World Network », Journal of Artificial Societies and Social Simulation, vol. 18, $\mathrm{n}^{\circ} 4,2015$, http://jasss.soc.surrey.ac.uk/18/4/12.html

Schumacher (Gijs), Vis (Barbara), « Why do social democrats retrench the welfare state? A simulation ", Journal of Artificial Societies and Social Simulation, vol. 15, $\mathrm{n}^{\circ}$ 3, 2012, http:// jasss.soc.surrey.ac.uk/15/3/4.html

Shutters (Shade), Hales (David), « Tag-Mediated Altruism is Contingent on How Cheaters Are Defined », Journal of Artificial Societies and Social Simulation, vol. 16, $\mathrm{n}^{\circ} 1,2013$, http:// jasss.soc.surrey.ac.uk/16/1/4.html

Smaldino (Paul), Epstein (Joshua), « Social conformity despite individual preferences for distinctiveness ", Royal society open science, Londres, Royal society publishing, mars 2015, http:// rsos.royalsocietypublishing.org/content/2/3/140437

Squazzoni (Flaminio), « The impact of agent-based models in the social sciences after 15 years of incursions ", History of Economic Ideas, vol. 18, n² 2, pp. 197-233, 2010 
Squazzoni (Flaminio), Casnici (Niccolò), « Is social simulation a social science outstation : a bilibiometric analysis of the impact of JASSS », Journal of Artificial Societies and Social Simulation, vol. 16, n 1, $2013 \mathrm{http}: / /$ jasss.soc.surrey.ac.uk/16/1/10.html

Squazzoni (Flaminio), « A social science-inspired complexity policy : beyond the mantra of incentivization », Complexity, vol. 19, n 6, pp. 5-13, 2014

Taghawi-Nejad (Davoud), « Modelling the Economy as an Agent-Based Process: ABCE, A Modelling Platform and Formal Language for ACE », Journal of Artificial Societies and Social Simulation, vol. 16, $\mathrm{n}^{\circ} 3$, 2013, http://jasss.soc.surrey.ac.uk/16/3/1.html

Takács (Károly), Squazzoni (Flaminio), « High Standards Enhance Inequality in Idealized Labor Markets ", Journal of Artificial Societies and Social Simulation, vol. 18, n 4, 2015, http:// jasss.soc.surrey.ac.uk/18/4/2.html

Thiele (Jan), Kurth (Winfried), Grimm (Volker), « Agent-based modelling : Tools for linking Netlogo and R », Journal of Artificial Societies and Social Simulation, vol. 15, n 3, 2012, http:// jasss.soc.surrey.ac.uk/15/3/8.html

Thompson (Nicholas), Berr (Patrick), « Contra Epstein : good explanations predicts », Journal of Artificial Societies and Social Simulation, vol. 12, $\mathrm{n}^{\circ}$ 1, $2009 \mathrm{http}: / /$ jasss.soc.surrey.ac.uk/12/1/9.html Venturini (Tomaso), Jensen (Pablo), Latour (Bruno), « Fill in the gap : toward a new alliance for social and natural sciences ", Journal of Artificial Societies and Social Simulation, vol. 18, $\mathrm{n}^{\circ} 2,2015$, http://jasss.soc.surrey.ac.uk/12/2/11.html

Von Randow (Gero), « When the centre becomes radical », Journal of Artificial Societies and Social Simulation, vol. 6, $\mathrm{n}^{\circ}$ 1, 2003 ; publication originale en allemand, Frankuter Allgemeine Sonntagszeitung, $\mathrm{n}^{\circ} 45$, p.43, nov. 2002, http://jasss.soc.surrey.ac.uk/6/1/5.html

Waldher (Annie), Wijermans (Nanda), « Communicating social simulation models to sceptical minds ", Journal of Artificial Societies and Social Simulation, vol. 16, $n^{\circ} 4,2013$, http:// jasss.soc.surrey.ac.uk/16/4/13.html

Weisbuch (Gérard), « From anti-conformism to extremism », Journal of Artificial Societies and Social Simulation, vol. 18, $\mathrm{n}^{\circ} 3,2015$, http://jasss.soc.surrey.ac.uk/18/3/1.html

Wilenski (Uri), Netlogo segregation model, Center for Connected Learning and Computer-Based Modeling, Northwestern University, Evanston, IL, 1997, http://ccl.northwestern.edu/netlogo/ models/Segregation

Woolridge (Michael), Jennings (Nicholas), « Pitfalls of agent-oriented development », Proceedings of the second international conference on autonomous agents, pp.385-391, New york, Association for Computing Machinery, 1998

Xie (Yu), Zhou (Xiang), « Modeling individual heterogeneity in racial residential segregation », Proceedings of the National Academy of Sciences, vol. 109, n² 29, pp. 11646-11651, 2012

\section{NOTES DE FIN}

1. Le premier numéro, sorti en janvier 1998, signe la naissance du champ autonome de la simulation sociale.

2. Amérindiens du sud-ouest de l'Amérique du Nord.

3. À l'exception notable des « sciences » économiques. 
4. Le « Manifesto of computational social science » (Conte et al., 2012) réclame rien moins que la fondation d'un CERN pour la simulation sociale, dont certains projets sont déjà largement financés par la commission européenne.

5. Evolution of Altruistic and Cooperative Habits, http://ccl.northwestern.edu/rp/each/index.shtml. 6. Les agents dit production rules associent des entrées à des sorties de façon plus ou moins complexe, typiquement par une simple équation.

7. Rodrigo Castro et Pablo Jakovkis (2015) fournissent de nombreux exemples, et décrivent notamment l'ambitieux Cybersyn, projet de modélisation de l'économie chilienne, interrompu en 1973 par le coup d'État de Pinochet.

INDEX

Mots-clés : code, interprétation, modélisation, simulation sociale, structuralisme

\section{AUTEUR}

\section{ARTHUR LEFÈVRE}

Doctorant en Sciences de l'information et de la communication, Université Paris 8 - Cemti 